\title{
Early metabolic response of breast cancer to neoadjuvant endocrine therapy: comparison to morphological and pathological response
}

Sarah Boughdad', Laurence Champion', Veronique Becette ${ }^{2}$, Pascal Cherel ${ }^{3}$, Emmanuelle Fourme ${ }^{4}$, Jerome Lemonnier ${ }^{5}$, Florence Lerebours ${ }^{6}$ and Jean-Louis Alberini ${ }^{1,7^{*}}$ (D)

\begin{abstract}
Background: Neoadjuvant endocrine therapy (NET) has shown efficacy in terms of clinical response and surgical outcome in postmenopausal patients with estrogen receptor-positive / HER2-negative breast cancer (ER+/HER2- BC) but monitoring of tumor response is challenging. The aim of the present study was to investigate the value of an early metabolic response compared to morphological and pathological responses in this population.

Methods: This was an ancillary study of CARMINA 02, a phase II clinical trial evaluating side-by-side the efficacy of 4 to 6 months of anastrozole or fulvestrant. Positron Emission Tomography/Computed Tomography using 2-deoxy-2$\left[{ }^{18}\right.$ F]fluoro-D-glucose (FDG-PET/CT) scans were performed at baseline (M0), early after 1 month of treatment (M1) and pre-operatively in 11 patients (74.2 yo \pm 3.6$)$. Patients were classified as early "metabolic responders" (mR) when the decrease of SUVmax was higher than 40\%, and "metabolic non-responders" (mNR) otherwise. Early metabolic response was compared to morphological response (palpation, US and MRI), variation of Ki-67 index, pathological response according to the Sataloff classification and also to Preoperative Endocrine Prognostic Index (PEPI) score. It was also correlated with overall survival (OS) and recurrence-free survival (RFS).

Results: Tumor size measured on US and on MRI was smaller in $\mathrm{mR}$ than $\mathrm{mNR}$, with the highest statistically significant difference at $\mathrm{M} 1$ ( $p=0.01$ and $7.1 \times 10^{-5}$, respectively). No statistically significant difference in the variation of tumor size between MO and M1 assessed on US or MRI was observed between $\mathrm{mR}$ and $\mathrm{mNR}$. mR had a better clinical response: no progressive disease in $\mathrm{mR}$ vs 2 in $\mathrm{mNR}$ and 2 partial response in $\mathrm{mR}$ vs 1 partial response in $\mathrm{mNR}$. One patient with a pre-operative complete metabolic response had the best pathological response. Pathological response did not show any statistically significant difference between $\mathrm{mR}$ and $\mathrm{mNR}$. $\mathrm{mR}$ had better OS and RFS (Kaplan-Meier $p=0.08$ and 0.06 , respectively). All cancer-related events occurred in mNR: 3 patients died, 2 of them from progressive disease.
\end{abstract}

Conclusions: FDG-PET/CT imaging could become a "surrogate marker" to monitor tumor response, especially as NET is a valuable treatment option in postmenopausal women with ER+/HER2- BC.

Keywords: Breast cancer, Neoadjuvant endocrine therapy, Estrogen receptor-positive / HER2-negative, FDG, PET/CT, Metabolic response, Morphological response, Pathological response

\footnotetext{
* Correspondence: jean-louis.alberini@curie.fr

${ }^{1}$ Department of Nuclear Medicine, Institut Curie-Saint-Cloud, 92210

Saint-Cloud, France

${ }^{7}$ Université Versailles Saint-Quentin, Paris-Saclay, Saint-Quentin-en-Yvelines,

France

Full list of author information is available at the end of the article
}

(c) The Author(s). 2020 Open Access This article is distributed under the terms of the Creative Commons Attribution 4.0 International License (http://creativecommons.org/licenses/by/4.0/), which permits unrestricted use, distribution, and reproduction in any medium, provided you give appropriate credit to the original author(s) and the source, provide a link to the Creative Commons license, and indicate if changes were made. The Creative Commons Public Domain Dedication waiver (http://creativecommons.org/publicdomain/zero/1.0/) applies to the data made available in this article, unless otherwise stated. 


\section{Introduction}

Neoadjuvant systemic therapies have been developed to achieve "tumor shrinkage" in locally advanced breast cancer in inoperable patients or to avoid radical mastectomy in patients with a tumor too large for primary breast-conserving surgery (BCS) [1] and to monitor tumor response. Nearly 70\% of breast cancers (BC) express hormone receptors and estrogen receptor-positive $(\mathrm{ER}+)$ and are less responsive to chemotherapy than ERnegative BC [2]. Neoadjuvant endocrine therapy (NET) is a recognized option of treatment for postmenopausal women with ER+/HER2- BC [3]. Pathological complete response (pCR) is the "gold standard" for evaluation of tumor response to neoadjuvant chemotherapy (NCT), as it is correlated with prognosis, although differences are observed among breast cancer subtypes [2]. However, pCR is uncommon after NET in ER+/HER2- BC [4] and is therefore not a suitable primary endpoint in NET clinical trials. Monitoring response to NET is challenging and, despite a poor reproducibility, clinical response is used as a primary endpoint in most clinical trials [5]. Monitoring of Ki67 index, a proliferation biomarker, has been increasingly used in NET clinical trials [5].

Imaging techniques such as breast ultrasound (US) [6] or MRI [7] have shown interesting results in neoadjuvant setting, but none of these techniques has been shown to be superior to clinical response in NET setting. Positron Emission Tomography/ Computed Tomography using 2deoxy-2- $\left[{ }^{18} \mathrm{~F}\right]$ fluoro-D-glucose (FDG-PET/CT) could therefore be a valuable tool to monitor in vivo changes in tumor glucose metabolism. FDG-PET/CT has shown efficacy for $\mathrm{BC}$ staging $[8,9]$, monitoring of tumor response to NCT [10] and detection of recurrence [11]. CARMINA 02 (NCT00629616, [12]) is a French phase II multicenter, randomized neoadjuvant trial evaluating side-by-side the efficacy of anastrozole and fulvestrant in postmenopausal patients with non-metastatic ER+/HER2- BC. The aim of the present study was to investigate the value of an early metabolic response on FDG-PET/CT after 1 month of NET compared to morphological response assessed by palpation and imaging with US and MRI, variation of Ki-67 index and pathological response. The predictive value of early metabolic response for prognosis, survival and patient management, in terms of BCS rate and adjuvant therapy, were defined as secondary objectives.

\section{Material and methods \\ Study design}

The present study was an ancillary study of CARMINA 02 prospective trial ([12], Fig. 1). The primary endpoint of this trial was the clinical response rate according to RECIST 1.0 criteria [12] assessed after 4 to 6 months in each treatment arm. Secondary endpoints were tumor response on US and MRI according to RECIST 1.0 criteria, baseline and ontreatment Ki-67 index, BCS rate, pathological response assessed by the Sataloff classification, with partial pathological response defined as TB and PCR as TA [13], and survival parameters, with overall survival (OS), recurrencefree survival (RFS) and PEPI score (Preoperative Endocrine Prognostic Index, [14]). Metabolic response based on FDGPET/CT was an optional secondary endpoint in CARMINA 02 trial [15] and is the object of the present study. Postmenopausal patients with ER+/HER2-, T2 to T4, N0 to N3, M0 breast cancer were randomized to receive anatrozole or fuvestrant for 4 to 6 months before surgery. Each center decided on adjuvant treatment according to the local policy. Patients with biopsy-proven BC underwent clinical, US and MRI examinations at baseline (M0), after 1 month of treatment (M1) and pre-operatively (Pre-op). Tumor response was defined on the longest tumor diameter on palpation,

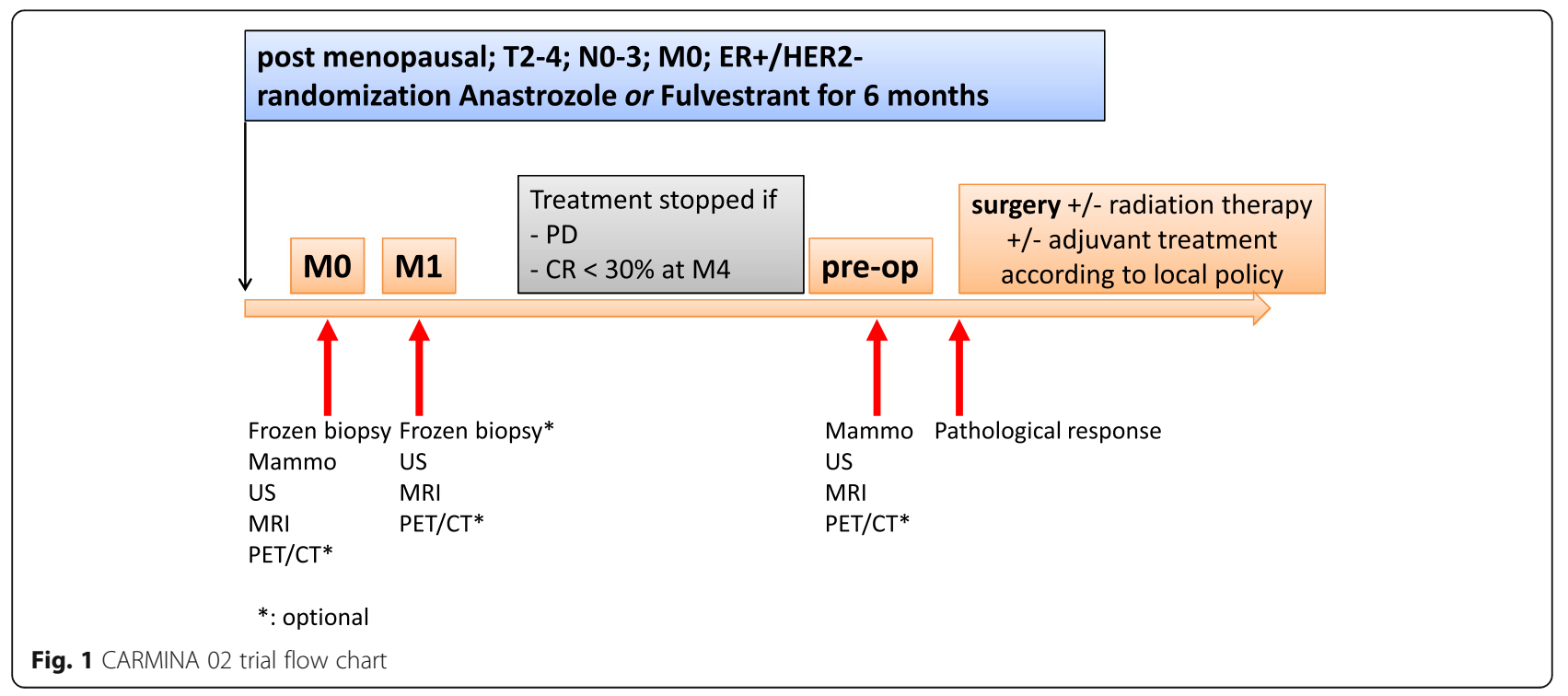


US and Dynamic Contrast-Enhanced (DCE)-MRI T1weighted sagittal slices. All radiological and pathological data were submitted to centralized expert review. An optional tumor biopsy was performed at M1 to assess variation of Ki-67 index. Patients had no lymph node involvement on palpation, US and MRI at MO. Patients underwent surgery at 4 months when tumor response on palpation was insufficient (stable or progressive disease). In patients with partial clinical response, NET was continued for an additional 2 months. Adjuvant therapy after surgery was decided by a multidisciplinary board. The study was conducted according to the Declaration of Helsinki and Good Clinical Practice guidelines and all patients provided their written informed consent. The study was authorized by the French Health Authority and approved by the Ethics Committee (Ile de France VIII).

\section{FDG-PET/CT imaging}

Patients underwent 3 serial FDG-PET/CT scans at M0, M1 and Pre-op in our institution from December 2007 to December 2010. Patients received an intravenous injection of $4-4.5 \mathrm{MBq} / \mathrm{kg}$ of 18-FDG in the arm opposite to the tumor, when capillary blood glucose level was less than $8 \mathrm{mmol} / \mathrm{L}$ and after fasting for at least $6 \mathrm{~h}$. Whole body imaging PET/CT scans (from the vertex to midthighs) were acquired from 60 to $80 \mathrm{~min}$ after FDG injection on a PET/CT scanner (Discovery LS, General Electric Healthcare, Waukesha, WI, USA) on a 3D mode with $5-7$ bed positions of $4-5$ min each. Non contrastenhanced $\mathrm{CT}$ images were acquired with the following parameters: $40 \mathrm{mAs}, 140 \mathrm{kV}, 5 \mathrm{~mm}$ section thickness, $0.8 \mathrm{~s}$ per CT rotation, $22.5 \mathrm{~mm} / \mathrm{s}$ table speed. This acquisition was used for attenuation correction, fusion, and also for diagnosis. Immediately after the CT, PET data were collected in a caudo-cranial direction. The CT data were resized from a $512 \times 512$ matrix to a $128 \times 128$ one, in order to match the PET data and to fuse the images. Images were analyzed by two nuclear medicine physicians on a Xeleris workstation (General Electric HealthCare) with triangulation tools for 3D vision.

\section{Images analysis}

SUVmax (Standard Uptake Value maximum) were measured by using a manually-delineated VOI (Volume of Interest) including the whole tumor. Early metabolic response was defined at M1 and late metabolic response was defined after at least 4 months of NET (Pre-op), in order to confirm the persistence of the metabolic response observed at M1. A cut-off of $40 \%$ for the SUVmax decrease (Delta-SUVmax) at M1 was used to differentiate 2 groups of patients: "metabolic responders" $(\mathrm{mR})$ and "metabolic non-responders" (mNR), according to results of a previously published study [15]. It was defined using a ROC analysis $(p=0.006)$.

\section{Survival analysis}

The correlation of early metabolic response with OS, RFS and PEPI score was studied. The PEPI score [16] combines pathological response (ypTN), Ki-67 index and ER Allred score and is relevant to predict RFS in NET setting [16].

\section{Statistical analysis}

Unpaired and two-sided Student tests were used to confirm significant differences in Delta-SUVmax at M1 and at Pre-op between $\mathrm{mR}$ and $\mathrm{mNR}$ according to the $40 \%$ Delta-SUVmax cut-off, and to compare morphological response (palpation, US and MRI) and variation of Ki-67 index at each time-point (M0, M1 and Pre-op or surgery for Ki-67) in $\mathrm{mR}$ and mNR. A Fisher's exact test was used to compare pathological response, PEPI score, BCS rate and adjuvant therapy (endocrine therapy or chemotherapy) between $\mathrm{mR}$ and $\mathrm{mNR}$. Differences in OS and RFS were analyzed using the Kaplan-Meier method. The influence of histological type (ductal or lobular) and the endocrine treatment arm (anastrozole or fulvestrant) on SUVmax was also analyzed using a Student test. The $p$ values considered as statistically significant were $<0.05$ and most statistical analysis were done using GraphpadPrism7.0b and R softwares.

\section{Results}

\section{Patient characteristics}

Among the 116 patients enrolled in the CARMINA 02 trial between 2007 and 2011, 11 patients (mean age \pm standard deviation: $74.2 \mathrm{y} \pm 3.6$; range: $67-87$ years) treated in our institution with 3 serial PET/CT scans available were included in the present study. All patients were clinically node negative. Patient baseline characteristics are presented in Table 1 and were comparable to those observed in the CARMINA 02 trial [14]. Seven of these 11 patients were randomized to anastrozole and 4 were randomized to fulvestrant. No significant differences in SUVmax according to treatment arm $(p=0.84,0.74$ and $p=0.71$ at M0; M1 and Pre-op, respectively) or histological type ( $45 \%$ lobular vs $55 \%$ ductal carcinoma; $p>0.05$ ) were observed, allowing global data analysis. Although tumor size was smaller in $\mathrm{mR}$, no difference of SUVmax at M0 was found between $\mathrm{mR}$ and $\mathrm{mNR}(p=0.41)$. According to the $40 \%$ Delta-SUVmax cut-off used at M1, 5 patients were classified as $\mathrm{mR}$ and 6 were classified as mNR (Figs. 2 and 3). Significant differences between $\mathrm{mR}$ and $\mathrm{mNR}$ were found at M1 ( $p=0.0002)$ and Pre-op ( $p=0.04)$ (Fig. 3).

\section{Morphological data}

Morphological and pathological responses are summarized on Table 2. Tumor sizes on US or MRI were different between $\mathrm{mR}$ and $\mathrm{mNR}$ at M0, M1 and Pre-op, with the better $p$-value for MRI. Although not statistically significant, 
Table 1 Baseline characteristics

\begin{tabular}{ll}
\hline Mean age \pm SD (years) & $74.2 \pm 3.6$ \\
Mean tumor size; range $(\mathrm{mm})$ & $47(30-70)$ \\
Tumor Stage & 9 \\
T2 & 2 \\
T3 & \\
Histological type & 6 \\
Ductal & 5 \\
Lobular & \\
Elston-Ellis Grade & 1 \\
Grade I & 9 \\
Grade II & 1 \\
Grade III & $12.4 \pm 8.5$ \\
Mean Ki-67 index (\%) & \\
ER Allred Score & 1 \\
6 & 2 \\
7 & 8 \\
8 &
\end{tabular}

differences in the variation of tumor size between M0 and M1 were observed between $\mathrm{mR}$ and $\mathrm{mNR}$ [palpation $(-1 \% \pm 12$ vs $-0.2 \% \pm 18.5 ; p=0.9)$, US $(-15.4 \% \pm 10.9$ vs $-2.7 \% \pm 22.8 ; p=0.26)$ and MRI $(-20.5 \% \pm 7.4$ vs $-9.4 \% \pm$ 16.8; $p=0.15)$, Fig. 4a,b,c]. Those differences persisted between $\mathrm{M0}$ and Pre-op [palpation $(-23.7 \% \pm 15.5$ vs $-8.4 \% \pm 21.7 ; p=0.21)$, US $(-28.4 \% \pm 22.2$ vs $-20.5 \% \pm$ 24.3; $p=0.6)$ and MRI $(-35.1 \% \pm 20.7$ vs $-18 \% \pm 21.2$; $p=0.21)$, Fig. 4a,b,c]. At Pre-op, a better clinical response was observed in $\mathrm{mR}$ : no progressive disease in $\mathrm{mR}$ vs 2 in $\mathrm{mNR}(p=0.4)$ and 2 partial response in $\mathrm{mR}$ vs 1 partial response in $\mathrm{mNR}$. Six patients were classified as having stable disease $(p=0.5)$. Two patients in each group had a partial response, as demonstrated by US or MRI and 7 patients were classified as having stable disease $(p=1)$.

\section{Pathological data}

No significant difference in Ki-67 index was observed between $\mathrm{mR}$ and $\mathrm{mNR}$ at M0 $(p=0.51), \mathrm{M} 1(p=0.23)$ and on surgical specimen $(p=0.41)$ (Table 2 ; Fig. $4 \mathrm{~d})$. Although not significant, mR had a lower Ki-67 index at M0, M1 and on surgical specimen, and a higher decrease of the Ki-67 index at M1 $(-61.5 \% \pm 20.9$ vs $+29 \% \pm 185 ; p=$ $0.21)$. No correlation between metabolic and pathological responses was found (Table 2), but pathological tumor size was significantly smaller in $\mathrm{mR}$ compared to $\mathrm{mNR}$ ( $22.8 \mathrm{~mm} \pm 6.1$ vs $42.5 \mathrm{~mm} \pm 10.8 ; p=0.0057)$. One patient with a complete metabolic response at Pre-op also presented the best pathological response (Sataloff TB), other patients were classified Sataloff TC (Table 2).

\section{Survival data}

Mean follow-up was $93.8 \pm 22.8$ months. Three mNR died during follow-up: 1 from glioblastoma and 2 from disease progression out of $3 \mathrm{mNR}$ with distant metastases ( 2 patients with bone metastases and 1 liver metastasis). $\mathrm{mR}$ had better OS and RFS, although not significant (Kaplan-Meier $p=0.08$ and 0.06, respectively; Fig. 5). The PEPI score was different between $\mathrm{mNR}$ and $\mathrm{mR}$, with a better prognostic index in $\mathrm{mR}(p=0.24$; Table 2$)$. More $\mathrm{mNR}$ received adjuvant chemotherapy than $\mathrm{mR}$ (67\% vs $20 \%$; $p=0.24$ ), because of a poor pathological response. No significant difference in BCS rate was observed between $\mathrm{mR}$ and $\mathrm{mNR}$, with 2 patients in each group $(p=1)$.

\section{Discussion}

Monitoring response to NET is challenging and no consensus has been reached concerning the best modality to use. Palpation and US are most commonly used in clinical practice, whereas breast MRI is increasingly used in clinical trials $[7,14]$. Mammography is not a valuable tool because it underestimates tumor size compared to the surgical specimen [17]. FDG-PET/CT imaging has shown high performances in the NCT setting $[18,19]$. Only one previously published study assessed the value of metabolic response to NET in 11 NO BC patients with a high expression of ER (ER Allred score 7-8) [15]. Because we have previously shown the prognostic value of metabolic response to endocrine treatment on Progression Free Survival in metastatic $\mathrm{BC}$ patients [20], we decided to prospectively assess the value of metabolic response to NET in a homogenous population of postmenopausal women with non-metastatic, ER+/HER2- BC included in a clinical trial. Performances of FDG-PET/CT imaging were compared with those of morphological and pathological parameters. In the Ueda's study [15], a 40\% DeltaSUVmax cut-off was determined using a ROC analysis and after 4 weeks of NET. Therefore, with the same delay of 4 weeks between baseline PET (M0) and assessing PET (M1), we used the same Delta-SUVmax cut-off to define early metabolic response. This cut-off allowed us to differentiate $\mathrm{mR}$ and $\mathrm{mNR}$ with a significant difference at M1 that persisted at Pre-op. We were also able to compare metabolic response with morphological response and with Ki-67. However, compared to the Ueda's study, in whom morphological imaging response was based on breast US alone, MRI data were incorporated in the morphological response criteria in the present study.

We found that patients with smaller tumors at diagnosis had a better pathological response in agreement with the literature [21]. $\mathrm{mR}$ had smaller tumors on morphological examinations, except on palpation which is known for poor reproducibility. In the present study, significant differences in tumor size decrease, assessed on US and MRI, 

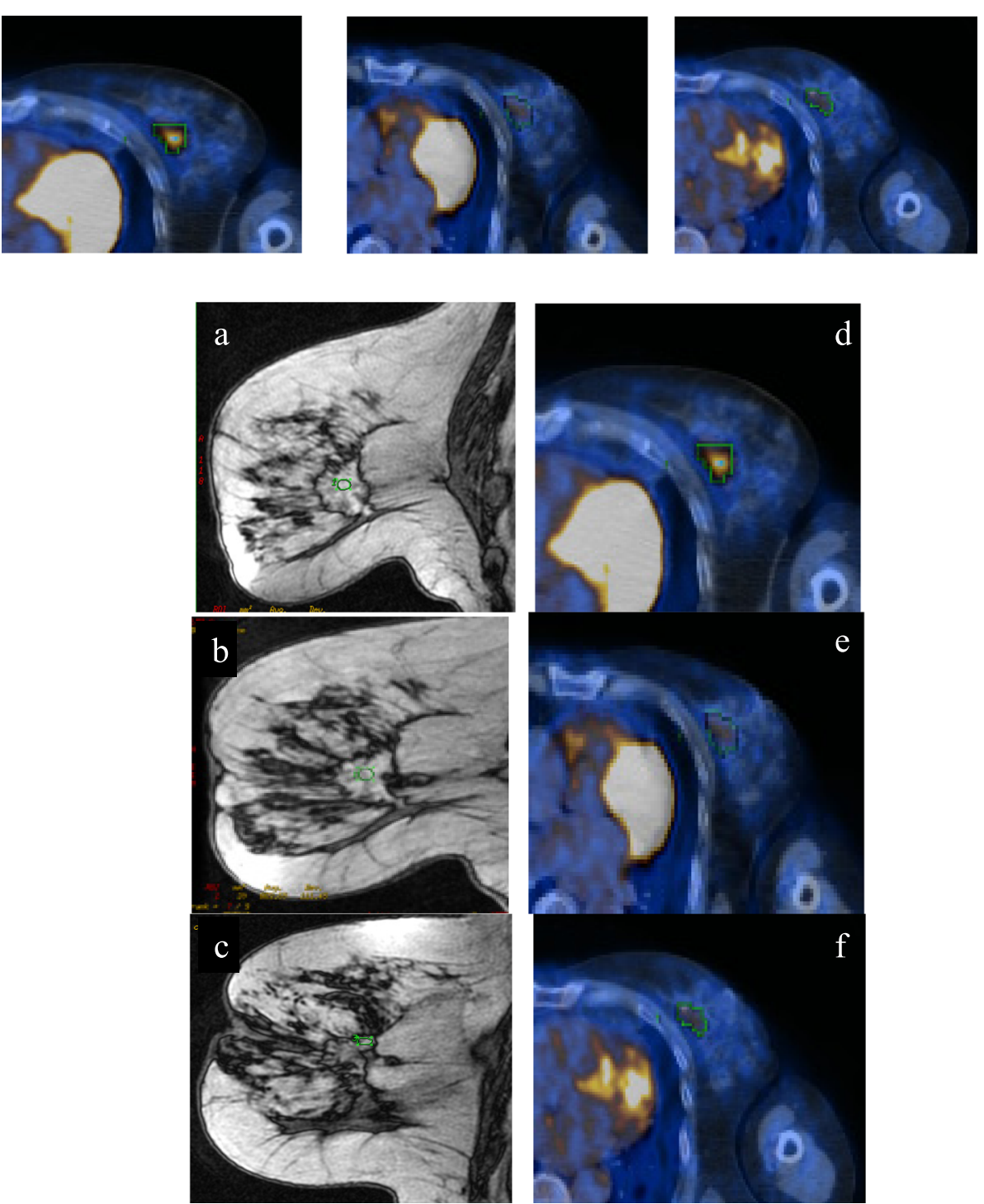

Fig. 2 Decrease of tumor size on gadolinium-enhanced, T1-weighted sagittal MRI slices at M0 (a, 30 mm), M1 (b, 25 mm) and Pre-op (c, 22 mm) in a 76 year-old $\mathrm{mR}$ patientMetabolic response was demonstrated on FDG-PET/CT fused axial slices at MO (d, SUVmax = 5), M1 (e, SUVmax = 3) and Pre-op (f, SUVmax = 3).

were observed between $\mathrm{mR}$ and $\mathrm{mNR}$ at M1 and at Preop (Table 2), as well as in the Ueda's study.

In the present study, although mNR had a higher Ki-67 index at each time-point compared to $\mathrm{mR}$, no significant difference in variation of Ki-67 index was observed between $\mathrm{mR}$ and $\mathrm{mNR}$ at $\mathrm{M} 1$ or on surgical specimen. A wide range of values for Ki-67 index was also observed in both groups, likely related to the small sample size of our cohort, which may have prevented the demonstration of significant differences. In CARMINA 02 trial, Ki-67 index was significantly reduced from the first month with both treatments (anastrozole or fulvestrant) and its level at the time of surgery was associated with pathological, but not with clinical response [14]. Ueda et al. compared metabolic response to variation of Ki-67 index at 2 weeks and 12 weeks, date of surgery, and found significantly higher decreases of Ki-67 index at 2 weeks and on surgical specimen in $\mathrm{mR}$ compared to $\mathrm{mNR}$ [15]. Monitoring of Ki-67 index has been increasingly used in NET trials [5]. However, no consensus has been reached concerning neither the scoring method, the interpretation nor the standard cut-off of Ki-67 index [22]. The IMPACT trial has shown that variation of Ki-67 index, assessed after 2 weeks of NET, was more predictive of RFS than the baseline value [5]. pCR is the "gold standard" for assessment of response of $\mathrm{BC}$ to NCT, as it is correlated with prognosis [2]. However, pCR is rarely observed after NET $[4,14]$. Moreover, there is no evidence at the present time that pCR constitutes a prognostic factor in NET 


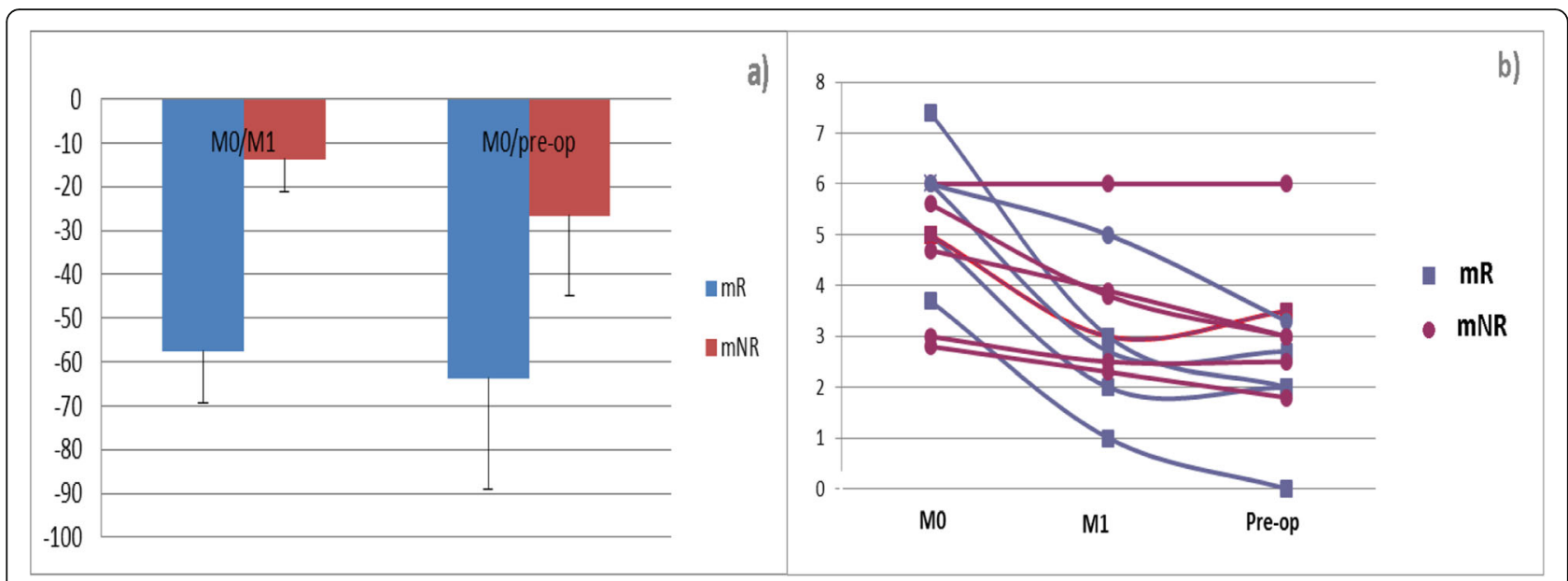

Fig. 3 Variation of mean Delta-SUVmax in $\mathrm{mR}$ and $\mathrm{mNR}(\mathbf{a})$ and of absolute SUVmax in each patient (b)

setting in contrast with NCT. In the present study, no patient achieved pCR (Sataloff TA), but one patient with a complete metabolic response at Pre-op achieved the best pathological response (Sataloff TB). Similarly, Ueda et al. did not find any pCR [15].

In terms of survival, all cancer-related events, such as distant metastases and BC-related deaths, occurred in $\mathrm{mNR}$ and a trend towards better OS and RFS was observed in $\mathrm{mR}$. A better prognosis according to PEPI score was observed in $\mathrm{mR}$. It is noteworthy that, in the
CARMINA 02 trial, PEPI score was the only variable significantly predictive of RFS [14].

The main limitation of the present study is the small number of patients included. According to Gebhart et al. [23], molecular imaging should be incorporated in translational research efforts. However, the present study illustrates the difficulty to include patients in imaging protocol in addition to a clinical trial. The fact that metabolic response based on FDG-PET/CT was an optional secondary endpoint in this trial was a critical

Table 2 Metabolic, morphological and pathological measurements at M1, Pre-op and on the surgical specimen. Student tests were used to compare metabolic, morphological data and Ki-67 data in mR and mNR. Fisher's exact tests were used for pathological data

Mo

$\begin{array}{ll} & \text { Clinical size }(\mathrm{mm}) \\ & \text { US size }(\mathrm{mm}) \\ & \text { MRI size }(\mathrm{mm}) \\ & \text { Ki-67 }(\%) \\ & \text { SUVmax }(\mathrm{g} / \mathrm{mL}) \\ & \text { Clinical size }(\mathrm{mm}) \\ & \text { US size }(\mathrm{mm}) \\ \text { Pre-op } & \text { MRI size }(\mathrm{mm}) \\ & \text { Number of patients } \\ & \text { Ki } 67(\%) \\ \text { BCS rate } & \text { Sataloff (TA + TB vs TC + TD) (n) } \\ \text { Surgical specimen } & \text { PEPI score (I + II vs III) (n) }\end{array}$

SUVmax $(\mathrm{g} / \mathrm{mL})$

Clinical size $(\mathrm{mm})$

US size (mm)

MRI size $(\mathrm{mm})$

Ki-67 (\%)

SUVmax $(\mathrm{g} / \mathrm{mL})$

Clinical size $(\mathrm{mm})$

$\mathrm{mR}(5 \mathrm{pts})$
$5.4 \pm 1.4$
$41 \pm 11.4$
$26.4 \pm 4.7$
$\mathbf{2 6} \pm \mathbf{3 . 7}$
$10.6 \pm 4.4$
$\mathbf{2 . 6} \pm 1.1$
$42.5 \pm 11.9$
$\mathbf{2 2 . 6} \pm \mathbf{6 . 3}$
$\mathbf{2 0 . 8} \pm \mathbf{4 . 2}$
$3.6 \pm 1.9$
$\mathbf{2} \pm 1.3$
$32 \pm 12.5$
$\mathbf{1 8 . 2} \pm \mathbf{7 . 3}$
$\mathbf{1 7 . 4} \pm \mathbf{6 . 7}$
2
$8.6 \pm 9.8$
1 vs 4
4 vs 1

$\mathrm{mR}$ (5 pts)

mNR (6 pts)

$4.7 \pm 1.5$

0.41

$52.5 \pm 8.8$

0.105

$40.3 \pm 11.6$

0.03

$44.7 \pm 8.3$

0.0016

$14 \pm 11.1$

0.51

$3.9 \pm 1.4$

0.00017

$51.7 \pm 7.5$

0.23

$34.2 \pm 2.4$

0.01

$39.7 \pm 4.8$

$7.1 \mathrm{E}-5$

$8.2 \pm 8$

0.23

$3.3 \pm 1.4$

0.018

$47.5 \pm 10.4$

0.06

$31.3 \pm 9.5$

0.041

$35.8 \pm 7.7$

0.002

2

$12.3 \pm 7.9$

0.41

0 vs 6

1

2 vs 4 


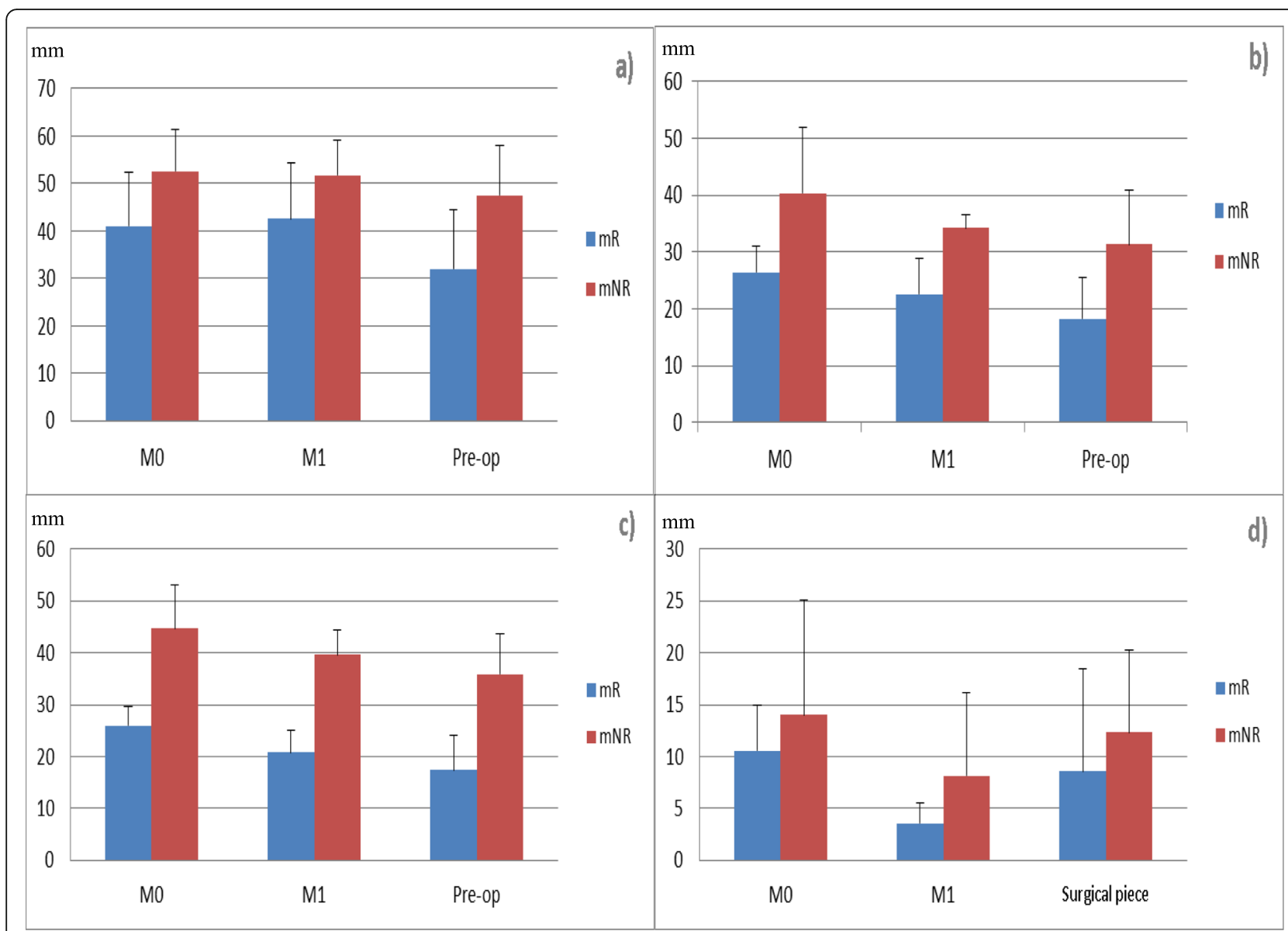

Fig. 4 Variations of tumor size (mm) assessed on palpation (a), US (b), MRI (c) and of Ki-67 index (\%) (d) at M0; M1 and Pre-op (a,b,c) or on surgical specimen (d) in $\mathrm{mR}$ and $\mathrm{mNR}$

limit and might explain that the rate of patients who underwent $3 \mathrm{PET} / \mathrm{CT}$ scans was as low as $10 \%$. We were able to recruit in a single center 11 patients from $87 \mathrm{~N} 0$ patients among the 116 one enrolled in this multicenter, randomized trial carried out between October 2007 and April 2011. The patients' age with a mean age of 74.2 $\mathrm{y} \pm 3.6$ was also a drawback for an easy recruitment to perform serial imaging exams.

Several limitations also concern the use of SUVmax in view of its marked variability and its failure to display tumor heterogeneity are well known but this parameter is widely used to monitor response of BC to NCT [19]. SUVmax has been shown to be useful in routine practice, as it is simple and reproducible and could be a valuable tool if FDG-PET/CT was validated in the NET setting [15]. Other semi-quantitative tools, such as Metabolic Tumor Volume, TLG (Total Lesion Glycolysis) or SUVpeak (average SUV value in a 10-voxel region including SUVmax) could be evaluated, although they have not been shown to be superior to SUVmax measurements and they have not been tested in monitoring response of BC to NET [24].
Tumor biopsies were generally performed after PET/CT scan. However, in 3 patients, they were performed before (1, 2 and 30 days, respectively). This action might generate inflammatory conditions which potentially induce increased FDG uptake and introduce a bias.

Almost one half of patients in this cohort presented with lobular carcinoma, which is more frequent in elderly patients [25], but no significant difference in SUVmax was observed according to histological type, probably due to the small number of patients. Glucose metabolism in lobular carcinoma has been shown to be lower than in ductal carcinoma [26] and should be taken into account in future studies together with the $\mathrm{BC}$ molecular subtype, which is also associated with variations in glucose metabolism [27]. The follow-up in this study could be considered to be relatively short. Longer follow-up is needed in this specific population of patients with low proliferative ER+/HER2- BC, who have a better prognosis than other $\mathrm{BC}$ molecular subtypes, but which may experience late relapses [28]. 


\section{Overall survival}

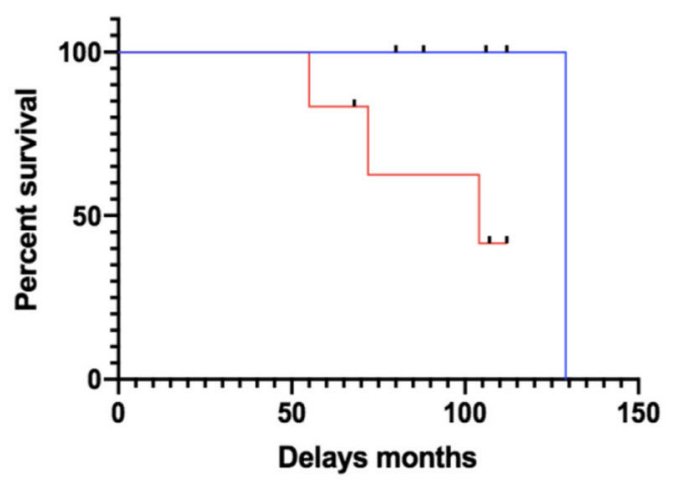

\section{+ Responders \\ - Non-Responders}

\section{Recurrence free survival}

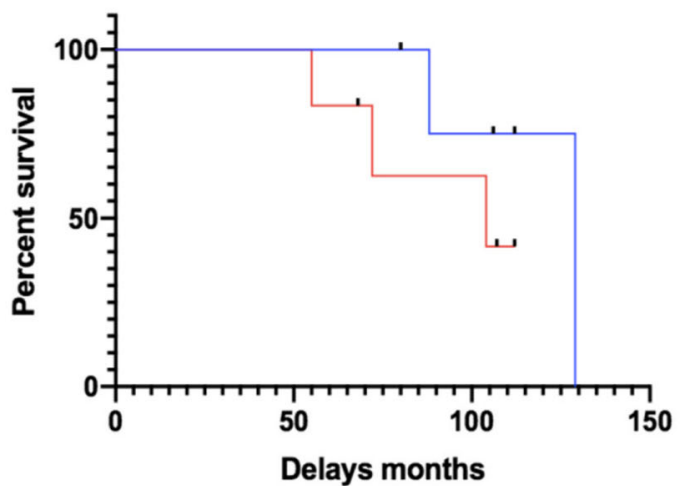

+ Responders

$\rightarrow$ Non-Responders

Fig. 5 Survival parameters, overall survival (OS) and recurrence-free survival (RFS) in mR and mNR using Kaplan-Meier method

18F-FES (fluoro-estradiol) targets ER and visualizes its functional in vivo pathway, which could predict response of $\mathrm{BC}$ to therapy and guide therapy selection for each patient. ER expression is a prerequisite to initiate endocrine therapy, but does not accurately reflect activation of the ER pathway in the tumor. 18F-FES and 18F-FDG have been shown to be complementary tools to monitor response of ER+ BC to NET [29, 30]. However, most studies involving 18F-FES have been performed in metastatic settings and further investigations are needed to define the value of 18F-FES in the NET setting.

\section{Conclusion}

Despite some limitations, this ancillary study of CARMINA 02 trial showed that early metabolic response can be more informative than morphological response and should be further investigated in a larger cohort of patients. If these results were confirmed, FDG-PET/CT could become a simple "surrogate marker" to monitor tumor response, especially as NET is a valuable treatment option in postmenopausal women with ER rich/HER2- BC. Assessment of early metabolic response allows adjustment of treatment, such as early "switch" to a more effective treatment option such as chemotherapy or targeted therapy, thereby improving patient care and prognosis.

\section{Abbreviations}

BC: Breast cancer; BCS: Breast conserving surgery; ER: Estrogen receptor; FDG: 2-deoxy-2-[18F]fluoro-D-glucose; mNR: Metabolic non-responders; mR: Early metabolic responders; MRI: Magnetic resonance imaging; NCT: Neoadjuvant chemotherapy; NET: Neoadjuvant endocrine therapy; OS: Overall survival; pCR: Pathological complete response; PEPI: Preoperative endocrine prognostic index; PET/CT: Positron emission tomography/ computed tomography; Pre-op: Pre-operatively; RFS: Recurrence-free survival; SUVmax: Standard uptake value maximum; US: Breast ultrasound

\section{Acknowledgements}

Not applicable.

Authors' contributions

$F L, E F, J L, J L A$ conceived and designed the study. FL contributed to patient recruitment. $S B, L C, E F, J L$ were involved in the patient data analysis. VB performed the histological examination of the breast. PC interpreted the MRI examination. SB, LC, FL, JLA wrote the manuscript. All authors read and approved the final manuscript.

\section{Funding}

Conception and design of the CARMINA 02 trial were funded by Unicancer (grant number: UCBG0609). UNICANCER received funds from La Ligue contre le Cancer and AstraZeneca. 


\section{Availability of data and materials}

The clinical data belong to the Sponsor UNICANCER. The data are available and can only be uploaded to a platform that would be willing to sign a transfer agreement with Unicancer, in conformity with the European Commission's Standard Contractual Clauses; In addition, data can only be accessed by experts that are identified in advance, to whom Unicancer should be able to grant a personal access.

\section{Ethics approval and consent to participate}

The study was conducted according to the 1964 Helsinki declaration and its later amendments or comparable ethical standards, and Good Clinical Practice guidelines. All patients provided their written informed consent. The study was authorized by the French Health Authority and approved by the Ethics Committee (lle de France VIII).

\section{Consent for publication}

For manuscripts containing any individual person's data in any form (including individual details, images or videos), consent to publish must be obtained from that person, or in the case of children, their parent or legal guardian.

\section{Competing interests}

The authors declare that they have no competing interests.

\section{Author details}

'Department of Nuclear Medicine, Institut Curie-Saint-Cloud, 92210 Saint-Cloud, France. ${ }^{2}$ Department of Pathology, Institut Curie, Saint-Cloud, France. ${ }^{3}$ Department of Radiology, Institut Curie, Saint-Cloud, France. ${ }^{4}$ Department of Statistics, Institut Curie, Saint-Cloud, France. ${ }^{5}$ UNICANCER, UCBG, Paris, France. ${ }^{6}$ Department of Medical Oncology, Institut Curie, Saint-Cloud, France. 'Université Versailles Saint-Quentin, Paris-Saclay, Saint-Quentin-en-Yvelines, France.

Received: 29 July 2019 Accepted: 13 January 2020

Published online: 28 January 2020

\section{References}

1. Fumagalli D, Bedard PL, Nahleh Z, Michiels S, Sotiriou C, Loi S, et al. A common language in neoadjuvant breast cancer clinical trials: proposals for standard definitions and endpoints. Lancet Oncol. 2012;13:e240-8.

2. Von Minckwitz G, Untch M, Blohmer J-U, Costa SD, Eidtmann H, Fasching PA, et al. Definition and impact of pathologic complete response on prognosis after neoadjuvant chemotherapy in various intrinsic breast cancer subtypes. J Clin Oncol. 2012;30:1796-804.

3. Coates AS, Winer EP, Goldhirsch A, Gelber RD, Gnant M, Piccart-Gebhart M, et al. Tailoring therapies-improving the management of early breast cancer: St Gallen international expert consensus on the primary therapy of early breast cancer 2015. Ann Oncol. 2015;26:1533-46.

4. Chia YH, Ellis MJ, Ma CX. Neoadjuvant endocrine therapy in primary breast cancer: indications and use as a research tool. Brit J Cancer. 2010;103:759-64.

5. Dowsett M. Biomarker changes during neoadjuvant anastrozole, tamoxifen, or the combination: influence of hormonal status and HER-2 in breast cancer-a study from the IMPACT trialists. J Clin Oncol. 2005;23:2477-92.

6. Hieken TJ, Harrison J, Herreros J, Velasco JM. Correlating sonography, mammography, and pathology in the assessment of breast cancer size. Am J Surg. 2001;182:351-4.

7. Marinovich M, Sardanelli F, Ciatto S, Mamounas E, Brennan M, Macaskill P, et al. Early prediction of pathologic response to neoadjuvant therapy in breast cancer: systematic review of the accuracy of MRI. Breast. 2012;21:669-77.

8. Groheux D, Cochet A, Humbert O, Alberini JL, Hindie E, Mankoff D. 18FDGPET/CT for staging and restaging of breast cancer. J Nucl Med. 2016; 57(Suppl 1):17S-26S.

9. Groves AM, Shastry M, Ben-Haim S, Kayani I, Malhotra A, Davidson T, et al. Defining the role of PET-CT in staging early breast cancer. Oncologist. 2012; 17:613-9.

10. Groheux D, Giacchetti S, Espié M, Rubello D, Moretti J-L, Hindié E. Early monitoring of response to neoadjuvant chemotherapy in breast cancer with 18F-FDG PET/CT: defining a clinical aim. Eur J Nucl Med Mol Imaging. 2010;38:419-25.
11. Champion L, Brain E, Giraudet AL, Stanc EL, Wartski M, Edeline V, et al. Breast cancer recurrence diagnosis suspected on tumor marker rising. Cancer. 2010;117:1621-9.

12. Eisenhauer E, Therasse P, Bogaerts J, Schwartz L, Sargent D, Ford R, et al. New response evaluation criteria in solid tumours: revised RECIST guideline (version 1.1). Eur J Cancer. 2009;45:228-47.

13. Sataloff DM, Mason BA, Prestipino AJ, Seinige UL, Lieber CP, Baloch Z. Pathologic response to induction chemotherapy in locally advanced carcinoma of the breast: a determinant of outcome. J Am Coll Surg. 1995;180:297-306.

14. Ueda S, Tsuda H, Saeki T, Omata J, Osaki A, Shigekawa T, et al. Early metabolic response to neoadjuvant letrozole, measured by FDG PET/CT, is correlated with a decrease in the Ki67 labeling index in patients with hormone receptor-positive primary breast cancer: a pilot study. Breast Cancer. 2010;18:299-308.

15. Lerebours F, Rivera S, Mouret-Reynier M-A, Alran S, Venat-Bouvet L, Kerbrat $P$, et al. Randomized phase 2 neoadjuvant trial evaluating anastrozole and fulvestrant efficacy for postmenopausal, estrogen receptor-positive, human epidermal growth factor receptor 2-negative breast cancer patients: results of the UNICANCER CARMINA 02 French trial. Cancer. 2016;122:3032-40.

16. Ellis MJ, Tao Y, Luo J, A'hern R, Evans DB, Bhatnagar AS, et al. Outcome prediction for estrogen receptor-positive breast cancer based on postneoadjuvant endocrine therapy tumor characteristics. J Natl Cancer Inst. 2008;100:1380-8.

17. Keune JD, Jeffe DB, Schootman M, Hoffman A, Gillanders WE, Aft RL. Accuracy of ultrasonography and mammography in predicting pathologic response after neoadjuvant chemotherapy for breast cancer. Am J Surg. 2010;199:477-84.

18. Groheux D, Majdoub M, Sanna A, Cremoux PD, Hindié E, Giacchetti S, et al. Early metabolic response to neoadjuvant treatment: FDG PET/CT criteria according to breast cancer subtype. Radiology. 2015;277:358-71.

19. Wang Y, Zhang C, Liu J, Huang G. Is 18F-FDG PET accurate to predict neoadjuvant therapy response in breast cancer? A meta-analysis. Breast Cancer Res Treat. 2012;131:357-69.

20. Mortazavi-Jehanno N, Giraudet AL, Champion L, Lerebours F, Stanc EL, Edeline $\mathrm{V}$, et al. Assessment of response to endocrine therapy using FDG PET/CT in metastatic breast cancer: a pilot study. Eur J Nucl Med Mol Imaging. 2011;39:450-60

21. Kaufmann M, Minckwitz GV, Mamounas EP, Cameron D, Carey LA, Cristofanilli $\mathrm{M}$, et al. Recommendations from an international consensus conference on the current status and future of neoadjuvant systemic therapy in primary breast cancer. Ann Surg Oncol. 2011;19:1508-16.

22. Dowsett M, Nielsen TO, A'hern R, Bartlett J, Coombes RC, Cuzick J, et al. Assessment of Ki67 in breast cancer: recommendations from the international Ki67 in breast cancer working group. J Natl Cancer Inst. 2011;103:1656-64.

23. Gebhart G, Lamberts LE, Wimana Z, Garcia C, Emonts P, Ameye L, et al. Molecular imaging as a tool to investigate heterogeneity of advanced HER2positive breast cancer and to predict patient outcome under trastuzumab emtansine (T-DM1): the ZEPHIR trial. Ann Oncol. 2016;27:619-24.

24. Hatt M, Groheux D, Martineau A, Espie M, Hindie E, Giacchetti S, et al. Comparison between 18F-FDG PET image-derived indices for early prediction of response to neoadjuvant chemotherapy in breast cancer. J Nucl Med. 2013:54:341-9.

25. Li Cl, Uribe DJ, Daling JR. Clinical characteristics of different histologic types of breast cancer. Brit J Cancer. 2005;93:1046-52.

26. Gil-Rendo A, Martínez-Regueira F, Zornoza G, García-Velloso MJ, Beorlegui C, Rodriguez-Spiteri N. Association between [18F] fluorodeoxyglucose uptake and prognostic parameters in breast cancer. Br J Surg. 2009;96:166-70.

27. Groheux D, Giacchetti S, Moretti JL, Porcher R, Espié M, Lehmann-Che J, et al. Correlation of high 18F-FDG uptake to clinical, pathological and biological prognostic factors in breast cancer. Eur J Nucl Med Mol Imaging. 2011;38:426-35.

28. Overmoyer B. Treatment with adjuvant endocrine therapy for early-stage breast cancer: is it forever? J Clin Oncol. 2015;33:823-8.

29. Kurland BF, Peterson LM, Lee $J H$, Schubert EK, Currin ER, Link JM, et al. Estrogen receptor binding (FES PET) and glycolytic activity (FDG PET) predict progression-free survival on endocrine therapy in patients with ER+ breast cancer. Clin Cancer Res. 2017;23:407-15.

30. Groheux D. 18F-Fluoroestradiol PET to predict the response to neoadjuvant treatment of luminal breast cancer. J Nucl Med. 2016;58:683.

\section{Publisher's Note}

Springer Nature remains neutral with regard to jurisdictional claims in published maps and institutional affiliations. 